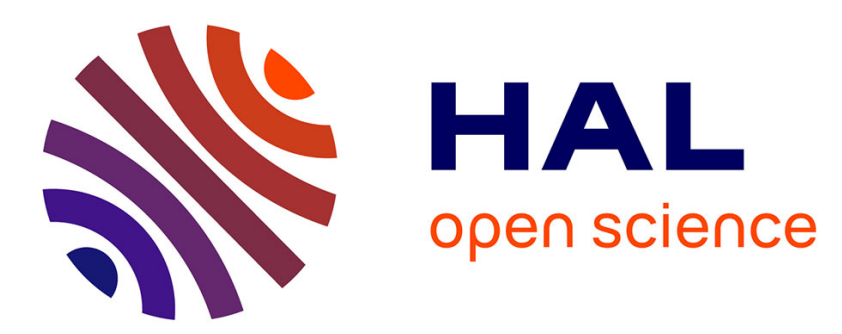

\title{
Fast-Calibration Reflectance-Transmittance Model to Compute Multiview Recto-Verso Prints
}

\author{
Serge Mazauric, Thierry Fournel, Mathieu Hébert
}

\section{To cite this version:}

Serge Mazauric, Thierry Fournel, Mathieu Hébert. Fast-Calibration Reflectance-Transmittance Model to Compute Multiview Recto-Verso Prints. Computational Color Imaging Workshop 2017, Mar 2017, Milan, Italy. pp.223-232, 10.1007/978-3-319-56010-6_19 . hal-01507469

\section{HAL Id: hal-01507469 \\ https://hal.science/hal-01507469}

Submitted on 13 Apr 2017

HAL is a multi-disciplinary open access archive for the deposit and dissemination of scientific research documents, whether they are published or not. The documents may come from teaching and research institutions in France or abroad, or from public or private research centers.
L'archive ouverte pluridisciplinaire HAL, est destinée au dépôt et à la diffusion de documents scientifiques de niveau recherche, publiés ou non, émanant des établissements d'enseignement et de recherche français ou étrangers, des laboratoires publics ou privés. 


\title{
Fast-calibration reflectance-transmittance model to compute multiview recto-verso prints
}

\author{
Serge Mazauric ${ }^{1}$, Thierry Fournel ${ }^{1}$, Mathieu Hébert ${ }^{1}$ \\ ${ }^{1}$ Univ Lyon, UJM-Saint-Etienne, CNRS, Institut d Optique Graduate School, Laboratoire \\ Hubert Curien UMR 5516, F-42023, Saint-Etienne, France \\ serge.mazauric@univ-st-etienne.fr
}

\begin{abstract}
Predicting simultaneously the spectral reflectance and transmittance of recto-verso prints is made easier thanks to a flux transfer matrix model. In the case where the printing support is symmetrical, i.e., its two sides are similar, the model can be calibrated from 44 halftone colors printed on the recto side, whose spectral reflectances and transmittance are measured. Color predictions are then allowed for any recto-verso halftone print illuminated on either side, and the inverse approach can be addressed: we can compute the digital layout for the recto and verso side that, once printed, can display different images according to the illuminated side.
\end{abstract}

Keywords: Computational printing, flux transfer models, duplex halftone print, multiview images.

\section{Introduction}

Spectral prediction models are key tools for fast and accurate color management in printing. They are also indispensable for designing advanced printing features such as those where the print displays different images according to the viewing conditions. Such multi-view effects can be obtained by using metallic inks [1] or a specular support [2] and observing the print in or out of the specular reflection direction. They can also be obtained by double-side printing with classical inks and supports, by observing one face in reflection mode or through both faces in transmission mode [3]. For these printing configurations, color management based on digital methods like ICC profile is almost impossible because the number of needed sample measurements, often beyond one thousand for single-mode observation, exponentially increases with the number of observation modes. The calibration time of the printing system thus becomes inacceptable, whereas a few tens of measurement often suffice to calibrate a prediction model, thus able to predict all reproducible colors in the different modes.

Among the most accurate models for the spectral reflectance of halftone prints, the physically-based models such as the Clapper-Yule model have high potential for extension to multilayer surfaces as they explicitly describe flux transfers between the layers. Some examples have been investigated in previous studies though stacks of printed

S. Mazauric, T. Fournel, M. Hébert, "Fast-Calibration Reflectance-Transmittance Model to Compute Multiview Recto-Verso Prints" In: Bianco S., Schettini R., Trémeau A., Tominaga S. (eds) Computational Color Imaging. CCIW 2017. Lecture Notes in Computer Science, vol. 10213, Springer, Cham, 223 - 232 (2017).

DOI: $10.1007 / 978-3-319-56010-6 \_19$ 
films [4] and double-side printed papers [5-6], configurations for which good prediction accuracy was achieve by means of acceptable number of calibration samples.

The main issues with these models where both reflectance and transmittance of prints are combined, it that they need a calibration for the reflectance mode, and a separate calibration for the transmittance mode: one parameter may have different values in the two modes. This issue was recently solved thanks to the so-called Duplex Primary Reflectance-Transmittance (DPRT) model, a two-flux transfer matrix model predicting simultaneously the reflectance and transmittance of duplex halftone prints on both faces, whose parameters related to the primaries are deduced from both reflectance and transmittance measurements and therefore optimal for the two modes [8]. In the general case where the two faces of the support are different or printed with different inks, the DPRT model needs 328 spectral measurements. In the present paper, we show that in the special case where the support is symmetric, i.e., its two faces are identical and printed with the same ink set, the number of spectral measurements for calibration is reduced to 104, while keeping the optimal predictive performances in reflectance and transmittance modes.

The corresponding model, called Double-Layer Reflectance-Transmittance model, relies on the idea of separating the printed support into two sublayers, in which spectral flux transfers are described. We propose to present it and to show a few example of multiview images that were designed by using the model in inversed mode.

\section{Two-flux transfer matrices}

A flux transfer model is particularly adapted to the layered structure of printed supports. When the printing support is strongly scattering, like paper or white polymer, we can use a two-flux model. The model presented in Ref. [8] applies with a stack of layers of strongly materials and the interfaces between them. They are characterized by four transfer factors: the front-side reflectance $r$, the back-side reflectance $r$ ', the forward transmittance $t$ and the backward transmittance $t$ '. The component is said to by symmetrical when $r=r$, and $t=t^{\prime}$. The angular distribution of the incident and transferred lights is to be considered in the definition of every transfer factor, which can be accordingly bi-hemispherical, directional-hemispherical, bi-directional and so on [10]. All fluxes and transfer factors may also depend upon wavelength.

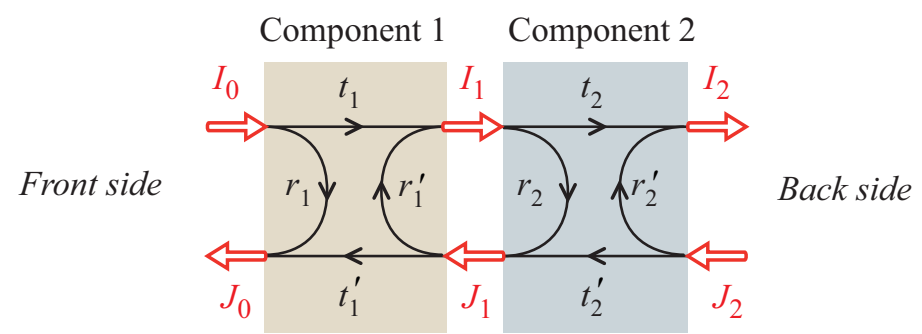

Fig. 1. Flux transfers between two flat components (arrows do not render orientation of light). 
When two components are on top of each other, interreflections of light occur between them, thus producing mutual exchanges between the fluxes propagating forwards (denoted as $I_{k}$ in Fig.1) and backwards (denoted as $J_{k}$ ). These exchanges can be easily described by using flux transfer matrices. For each component $k=1$ or 2 , assuming $t_{k} \neq 0$, the relations between incoming and outgoing fluxes is described by the following matrix equation:

$$
\left(\begin{array}{c}
I_{k-1} \\
J_{k-1}
\end{array}\right)=\frac{1}{t_{k}}\left(\begin{array}{cc}
1 & -r_{k}^{\prime} \\
r_{k} & t_{k} t_{k}^{\prime}-r_{k} r_{k}^{\prime}
\end{array}\right)\left(\begin{array}{c}
I_{k} \\
J_{k}
\end{array}\right),
$$

where the matrix is the transfer matrix attached to the component $k$, denoted as $\mathbf{M}_{k}$

$$
\mathbf{M}_{k}=\frac{1}{t_{k}}\left(\begin{array}{cc}
1 & -r_{k}^{\prime} \\
r_{k} & t_{k} t_{k}^{\prime}-r_{k} r_{k}^{\prime}
\end{array}\right)
$$

Grouping components 1 and 2 together, Eq. (1) can be repeated twice. One obtains:

$$
\left(\begin{array}{l}
I_{0} \\
J_{0}
\end{array}\right)=\mathbf{M}_{1}\left(\begin{array}{l}
I_{1} \\
J_{1}
\end{array}\right)=\mathbf{M}_{1} \mathbf{M}_{2}\left(\begin{array}{l}
I_{2} \\
J_{2}
\end{array}\right)=\mathbf{M}\left(\begin{array}{l}
I_{2} \\
J_{2}
\end{array}\right)
$$

where $\mathbf{M}$, product of the transfer matrices of the individual components, is the transfer matrix representing the two layers together, similarly defined as Eq. (2) in terms of its transfer factors $R, T, R^{\prime}$ and $T^{\prime}$. The multiplicative property of transfer matrices is true for any number of components, and the left-to-right position of the matrices in the product reproduces the front-to-back position of the corresponding components. Every transfer matrix has the structure displayed in Eq. (2) and from a given transfer matrix $\mathbf{M}=\left\{m_{i j}\right\}$, provided $m_{11} \neq 0$, one retrieves the transfer factors in the following way:

$$
\begin{aligned}
R & =m_{21} / m_{11}, \\
T & =1 / m_{11}, \\
R^{\prime} & =-m_{12} / m_{11}, \\
T^{\prime} & =m_{22}-m_{21} m_{12} / m_{11} .
\end{aligned}
$$

\section{Double-layer Reflectance-Transmittance (DLRT) model}

We will use flux transfer matrices to describe the reflection and transmission of light by recto-verso halftone prints. Let us first consider the support alone, by assuming it symmetrical and strongly scattering. Its structure is shown in Fig. 2: an effective diffusing medium of refractive index 1.5 is bordered by interfaces with the surrounding air. Since it is symmetrical, it has similar transfer factors $R(\lambda)$ and $T(\lambda)$ on its two sides, and is therefore represented by a transfer matrix, defined for each wavelength as:

$$
\mathbf{P}=\frac{1}{T}\left(\begin{array}{cc}
1 & -R \\
R & T^{2}-R^{2}
\end{array}\right),
$$




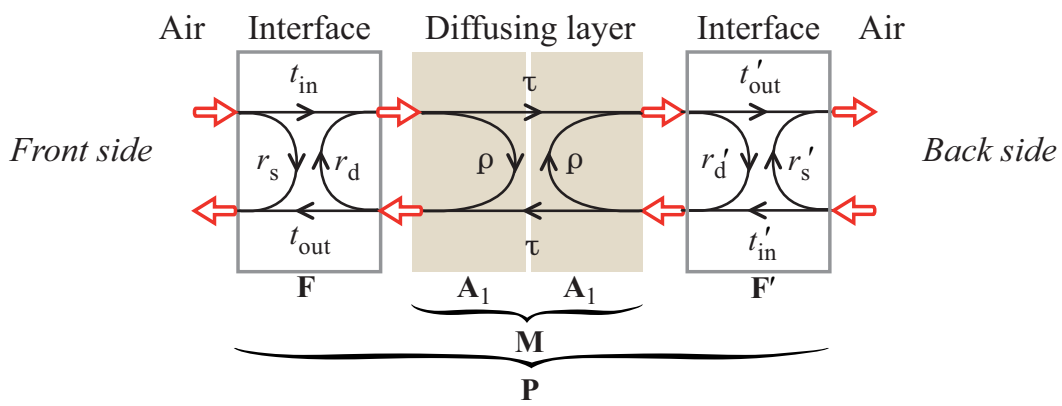

Fig. 2. Flux transfers between a diffusing layer (split into two identical sublayers) and its bordering interfaces, and corresponding flux transfer matrices.

The diffusing layer (considered without the interfaces) also has similar transfer factors $\rho(\lambda)$ and $\tau(\lambda)$ on its two sides. It is represented by the following transfer matrix:

$$
\mathbf{M}=\frac{1}{\tau}\left(\begin{array}{cc}
1 & -\rho \\
\rho & \tau^{2}-\rho^{2}
\end{array}\right) .
$$

The reflectances and transmittances of the interfaces depend on the measuring geometry. In the present study, measurements were done with the X-rite Color i7 spectrophotometer relying on the di: $8^{\circ}$ geometry in reflection mode (illumination with perfectly diffuse light over the hemisphere, and observation at $8^{\circ}$ from the normal of the sample by including the specular reflection component), and the d: $0^{\circ}$ geometry in transmission mode (illumination with perfectly diffuse light over the hemisphere, and observation in the normal of the sample). The corresponding transfer factors are given in Fig. 2. For these geometries and the refractive index value 1.5, the transfer matrices representing the interfaces at the recto and verso sides are given by:

$$
\begin{aligned}
& \mathbf{F}=\frac{1}{t_{\text {in }}}\left(\begin{array}{cc}
1 & -r_{d} \\
r_{s} & t_{\text {in }} t_{\text {out }}-r_{s} r_{d}
\end{array}\right)=\left(\begin{array}{cc}
1.111 & -0.667 \\
0.044 & 0.403
\end{array}\right), \\
& \mathbf{F}^{\prime}=\frac{1}{t_{\text {out }}}\left(\begin{array}{cc}
1 & -r_{s} \\
r_{d} & t_{\text {in }} t_{\text {out }}-r_{s} r_{d}
\end{array}\right)=\left(\begin{array}{cc}
1.111 & -0.044 \\
0.667 & 0.403
\end{array}\right),
\end{aligned}
$$

In practice, the measurable quantities are the spectral reflectance $R(\lambda)$ and transmittance $T(\lambda)$ of the support with interfaces, therefore matrix $\mathbf{P}$. The transfer matrix $\mathbf{M}$ for the diffusing layer without interfaces is then given by:

$$
\mathbf{M}=\mathbf{F}^{-1} \cdot \mathbf{P} \cdot \mathbf{F}^{-1} .
$$

Later, it will be convenient to decompose this diffusing layer into two similar sublayers (which justifies the appellation "double-layer reflectance-transmittance' model), thus represented by the transfer matrix:

$$
\mathbf{A}_{1}=\mathbf{M}^{1 / 2}=\left(\mathbf{F}^{-1} \cdot \mathbf{P} \cdot \mathbf{F}^{-1}\right)^{1 / 2}
$$


We can extend the model to the Neugebauer primaries, obtained when superposing cyan, magenta and yellow halftone screens: white (surface with no ink, labelled $i=1$ ) cyan, magenta, yellow, red (magenta + yellow), green (cyan + yellow), blue (cyan + magenta) and black (cyan + magenta + yellow). It is classical after Clapper and Yule [15] to consider the inked support as a four-component structure: the two interfaces, the ink layer and the diffusing layer. We proposed in [8] a different approach where the inked support without interfaces is one non-symmetrical layer of effective medium. According to the double-layer approach, we can then split it into two sublayers: one on the front side containing the inks represented by a matrix $\mathbf{A}_{i}$, one with the matrix $\mathbf{A}_{1}$ given by (9) for the non-inked back side. Thus, the transfer matrix representing the support covered by primary $i$ on the recto side only is written:

$$
\mathbf{P}_{i 1}=\mathbf{F} \cdot \mathbf{A}_{i} \cdot \mathbf{A}_{1} \cdot \mathbf{F}^{\prime}
$$

We thus have, for each primary $i=2, \ldots, 8$ :

$$
\mathbf{A}_{i}=\mathbf{M}_{i 1} \cdot \mathbf{A}_{1}^{-1}=\left(\mathbf{F}^{-1} \cdot \mathbf{P}_{i 1} \cdot \mathbf{F}^{\prime-1}\right) \cdot\left(\mathbf{F}^{-1} \cdot \mathbf{P}_{11} \cdot \mathbf{F}^{-1}\right)^{-1 / 2} \equiv \frac{1}{\tau_{i}}\left(\begin{array}{cc}
1 & -\rho_{i}^{\prime} \\
\rho_{i} & \tau_{i} \tau_{i}^{\prime}-\rho_{i} \rho_{i}^{\prime}
\end{array}\right)
$$

The transfer factors of the front sublayer, denoted $\rho_{i}, \rho_{i}^{\prime}, \tau_{i}$, and $\tau_{i}^{\prime}$, are obtained from (10) by applying formulas (4). Since the support is symmetrical, and the verso side is printed with the same inks as the recto side, we can create similar matrix as $\mathbf{A}_{i}$ for the verso side, by permuting $\rho_{i}$ and $\rho_{i}^{\prime}$ on the one hand, and $\tau_{i}$ and $\tau_{i}^{\prime}$ on the other hand, and finally replacing $i$ with $j$ :

$$
\mathbf{A}_{j}^{\prime}=\frac{1}{\tau_{j}^{\prime}}\left(\begin{array}{cc}
1 & -\rho_{j} \\
\rho_{j}^{\prime} & \tau_{j} \tau_{j}^{\prime}-\rho_{j} \rho_{j}^{\prime}
\end{array}\right)
$$

When a primary $i$ is on the recto side and a primary $j$ on the verso side, the matrix representing the whole inked support (interfaces excluded) is simply given by:

$$
\mathbf{M}_{i j}=\mathbf{A}_{i} \cdot \mathbf{A}_{j}^{\prime}
$$

The model can be extended to halftone colors (Fig. 3 ). We denote as $a_{i}(i=1, \ldots, 8)$ and $a_{j}^{\prime}(j=1, \ldots, 8)$ the surface coverages of the primaries on the recto side, respectively on the verso side. They are obtained from the surface coverages of the cyan, magenta and yellow inks using Demichel's equations, valable in case of typical stochastic or cluster dot halftoning techniques [16]. We use the transfer factors $\rho_{i}, \rho_{i}^{\prime}, \tau_{i}$, and $\tau_{i}^{\prime}$ issued from (10) and (4), and combine them according to the ink surface coverages $a_{i}$ and $a_{j}^{\prime}$ present on each side of the support. By using the generic letter $\xi$ for either $\rho$, $\rho$ ', $\tau$, or $\tau$ ', we have, for the recto side:

$$
\xi_{\left\{a_{i}\right\}}=\sum_{i=1}^{8} a_{i} \xi_{i 1}
$$

and for the verso side:

$$
\xi_{\left\{a_{i}^{\prime}\right\}}=\sum_{i=1}^{8} a_{i} \xi_{i 1}^{\prime}
$$




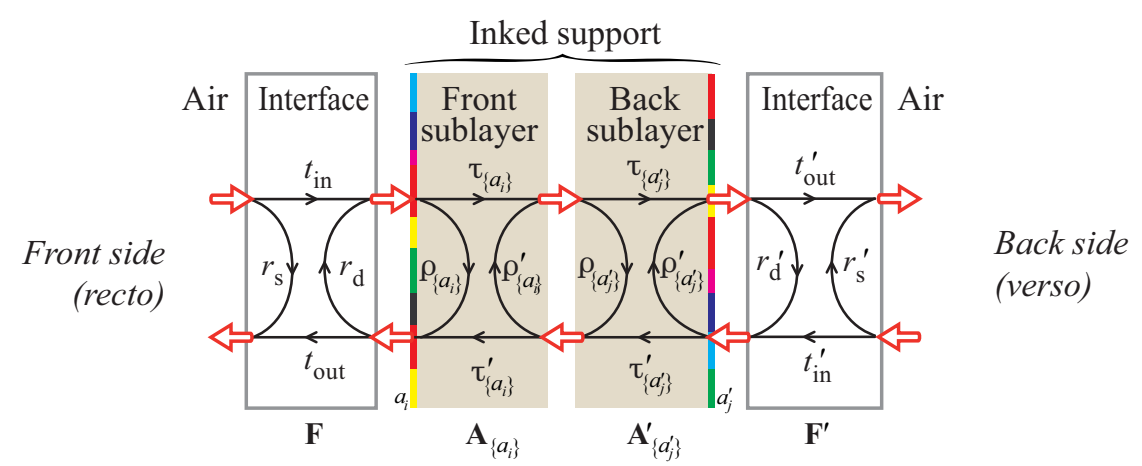

Fig. 3. Flux transfers within a homogeneous support printed with halftone on both sides: the inked support is split into two sublayers.

From these transfer factors given by (13) and (14), we create the transfer matrices representing the sublayers on the recto, respectively on the verso:

$$
\begin{aligned}
\mathbf{A}_{\left\{a_{i}\right\}} & =\frac{1}{\tau_{\left\{\mathrm{a}_{i}\right\}}}\left(\begin{array}{cc}
1 & -\rho_{\left\{\mathrm{a}_{i}\right\}}^{\prime} \\
\rho_{\left\{\mathrm{a}_{i}\right\}} & \left.\tau_{\left\{\mathrm{a}_{i}\right\}} \tau_{\left\{\mathrm{a}_{i}\right\}}^{\prime}-\rho_{\left\{\mathrm{a}_{i}\right\}} \rho_{\left\{\mathrm{a}_{i}\right\}}^{\prime}\right\}
\end{array}\right), \\
\mathbf{A}_{\left\{a_{j}^{\prime}\right\}}^{\prime} & =\frac{1}{\tau_{\left\{a_{j}^{\prime}\right\}}}\left(\begin{array}{cc}
1 & -\rho_{\left\{a_{j}^{\prime}\right\}}^{\prime} \\
\rho_{\left\{a_{j}^{\prime}\right\}} & \left.\tau_{\left\{a_{j}^{\prime}\right\}} \tau_{\left\{a_{j}^{\prime}\right\}}^{\prime}-\rho_{\left\{a_{j}^{\prime}\right\}} \rho_{\left\{a_{j}^{\prime}\right\}}^{\prime}\right\}
\end{array}\right)
\end{aligned}
$$

Finally, the duplex print with interfaces is represented by the transfer matrix:

$$
\mathbf{M}_{H}=\mathbf{F} \cdot \mathbf{A}_{\left\{a_{i}\right\}} \cdot \mathbf{A}_{\left\{a_{j}^{\prime}\right\}}^{\prime} \cdot \mathbf{F}^{\prime}
$$

and its reflectances and transmittances are deduced from this matrix by using (4).

Note that we can also predict, without further calibration effort, the spectral reflectances and transmittances of a doubled printing support. The matrix $\mathbf{M}_{H}$ is simply computed as follows:

$$
\mathbf{M}_{H}=\mathbf{F} \cdot \mathbf{A}_{\left\{a_{i}\right\}} \cdot \mathbf{A}_{1} \cdot \mathbf{F}^{\prime} \cdot \mathbf{F} \cdot \mathbf{A}_{1} \cdot \mathbf{A}_{\left\{a_{j}^{\prime}\right\}}^{\prime} \cdot \mathbf{F}^{\prime}
$$

\section{Calibration and verification of the model}

The DPRT model introduced in [8], valid for any non-symmetrical diffusing support, needed $8 \times 8=64 \mathrm{CMY}$ patches for its calibration, corresponding to the different combinations of the eight primaries on the front side and the eight primaries on the back side. The present version, valid for homogenous diffusing supports, needs only 8 rectoonly samples if the support is symmetrical and printed with the same set of inks on both sides. Since the four transfer factors of each sample must be measured, this makes an appreciable gain of time for the calibration of the DPRT model. The calibration of the model is made in two steps. 
The first step is the determination of the matrices $\mathbf{A}_{i}$, given by (10), for the eight Neugebauer primaries. For primary 1, the reflectance and transmittance of the unprinted support are measured on the recto side; for the other seven primaries, we print each primary with a coverage of $100 \%$ on the recto side, and measure the reflectances and transmittances of the printed support on its both sides. The reflectances and transmittances $\rho_{i}, \rho_{i}^{\prime}, \tau_{i}$, and $\tau_{i}^{\prime}$ for the front-side sublayer printed with each primary $i$ are computed, then the matrices $\mathbf{A}_{j}$ representing the back-side sublayer printed with each primary $j$.

The second step is establishing the correspondence between the nominal ink surface coverages (those specified in the digital layout) and the effective ones, observed on the printed paper through the spectral reflectance and transmittance. We follow the very efficient method introduced by Hersch et al. [7], also detailed in [16], which yields 12 curves computed from 36 single-ink halftones printed on the recto side. For each halftone, the effective ink surface coverages is estimated by minimizing the difference between the measured and predicted spectra, according to a metric which may be the rms deviation between the spectral reflectances and/or transmittances, or the equivalent color distance (e.g. CIELAB $\Delta \mathrm{E}_{94}$ computed from the spectra for a given illuminant and a given white reference).

Finally, for any recto-verso halftone defined by its nominal ink surfaces coverages (CMY values) on the recto and verso sides, the primary surface coverages $a_{i}$ and $a_{i}^{\prime}$ are computed according to the Demichel equations, the transfer matrix $\mathbf{M}_{H}$ of the rectoverso print is obtained by using equations (13) to (16), and the reflectances and transmittances by using formulas (4).

The model was verified over tens of recto-verso samples with CMY values randomly selected over the color gamut on both sides of the support. They were printed with both inkjet and electrophotography (laserjet) printer, each time on both high-quality supercalendered paper and usual office paper. The number of patches in each set is specified in Table 1. The prediction accuracy, in reflectance or transmittance mode, is assessed by the average CIELAB $\Delta E_{94}$ color distance between the predicted and measured spectra (see computation details in [16]).

Table 1. Average CIELAB $\Delta \mathrm{E}_{94}$ value between predicted and measured spectra

\begin{tabular}{llllccc}
\hline \hline Support & Printer & Set & Mode & \multicolumn{3}{c}{ Model } \\
\hline \hline \multirow{3}{*}{$\begin{array}{l}\text { Supercalen- } \\
\text { dered paper }\end{array}$} & \multirow{2}{*}{ Inkjet } & \multirow{2}{*}{ 36 samples } & Reflectance & 0,76 & 0,92 & 0,7 \\
& & & Transmittance & 0,75 & 1,01 & 0,94 \\
\cline { 4 - 7 } & Laserjet & 25 samples & Reflectance & 1,43 & 1,52 & 1,5 \\
& & & Transmittance & 0,87 & 1,5 & 1,18 \\
\hline Office paper & \multirow{2}{*}{ Inkjet } & \multirow{2}{*}{ 28 samples } & Reflectance & 1,31 & 1,06 & 1,1 \\
& & & Transmittance & 1,17 & 1,58 & 1,28 \\
\cline { 4 - 7 } & Laserjet & 25 samples & Reflectance & 1,39 & 1,27 & 1,35 \\
& & & Transmittance & 0,89 & 2,31 & 1,38 \\
\hline \hline
\end{tabular}

The values presented in Table 1 show that the prediction accuracy is rather good, even though slightly poorer with the laserjet printer due to a low printing homogeneity, 
and with the office paper which is also less homogeneous than the supercalendered one. In most cases, the DLRT model is more accurate, and even much more accurate in transmittance mode, than previously proposed models: the Duplex Clapper-Yule model (DCY) [8], and the Yule-Nielsen model ([7], and [6] for the extension to transmittance). Recall that the model is calibrated from only 44 recto-only color patches measured in reflectance and transmittance mode, and then that billions of recto-verso colors can be predicted.

\section{$5 \quad$ Multiview images}

Once the model is calibrated and its prediction accuracy has been verified, we can use it in an inversed mode to produce multiview prints, i.e., prints displaying different images according to the illumination and observation configurations. For example, we can illuminate the print on the observer's side (reflection mode) or the opposite side (transmission mode). We can also consider a transmission mode where ambient light slightly illuminates the observer's side (with similar SPDs on both sides), which corresponds to an addition of $\alpha$ times the transmittance and $(1-\alpha)$ times the reflectance, a configuration referred to as " $\alpha$-transmission mode".

The ink quantities to print on the recto and verso sides are obtained by performing a brute-force optimization in order to obtain the targeted CIE $1976 \mathrm{~L}^{*} \mathrm{a} * \mathrm{~b}^{*}$ values, or the targeted spectral radiance, in the two selected illumination modes. We generally compute first the ink surface coverages on the recto side yielding the reflection-mode image, then the ones on the verso side so as to obtain the targeted image in $\alpha$-transmission mode. The selection of the targeted colors for the two modes is critical since color matching is not always possible, a question addressed in [20].
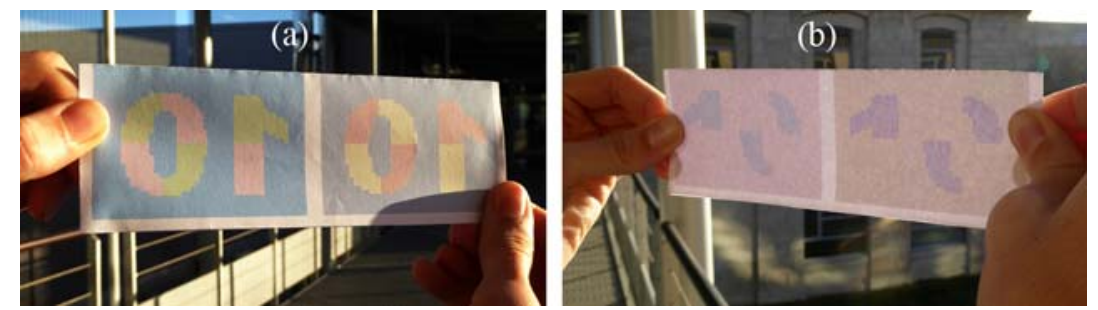

Fig. 4. Multiview recto-verso halftone print produced by laserjet printing on doubled office paper, displaying different images (a) on the verso side in reflection mode, and (b) on the recto side in transmission mode, under sunlight.

Fig. 4 shows an example of multi-view image printed by laserjet printer on doubled office paper. It displays (flipped) "10" patterns on the verso side is reflectance mode, and only part of these patterns remain visible in 0.9-transmittance mode, because the color of the other part matches the color of the background of the image. A second print is shown in Fig. 5, where randomly distributed squares of eight different colors are printed on the recto side, eight colors are correspondingly printed on the verso side in 
order to display, in 0.9-transmission mode, two patterns of homogenous color and a background with more contrasted texture. Registration of the recto and verso images, and perfect parallelism of the two paper sheet is crucial for a good rendering of the targeted visual effect.
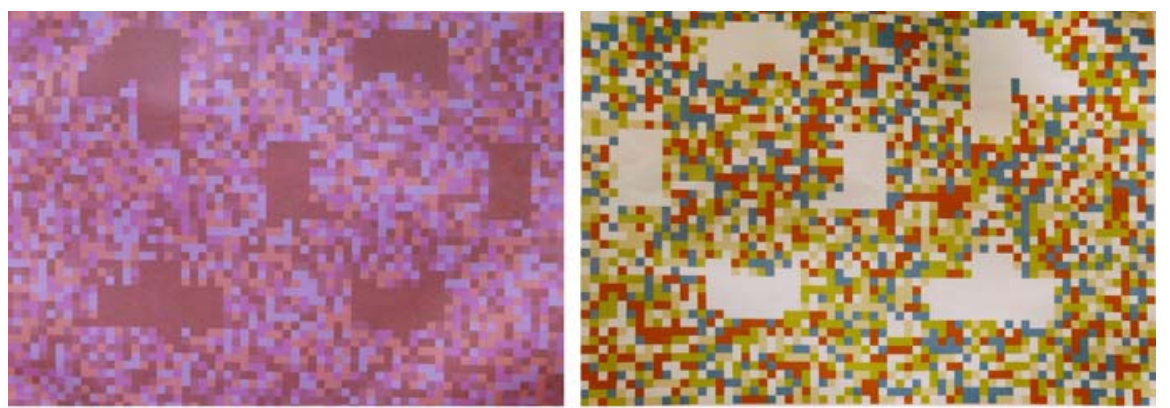

(a)

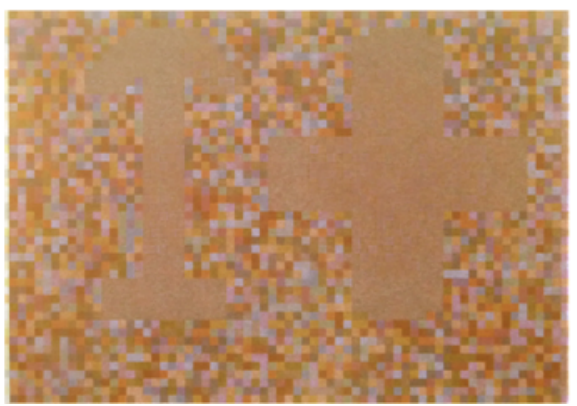

(b)

(c)

Fig. 5. Multiview recto-verso halftone print produced by laserjet printing on doubled office paper, displaying a mosaic of randomly distributed color squares on the recto side (a) and verso side (b), and homogenously colored patterns surrounded by a contrasted color mosaic in 0.9 transmission mode (c, with increased lightness).

\section{Conclusions}

The DLRT model is a two-flux transfer matrix model that allows the prediction of the reflectance and transmittance of duplex halftone prints with a better accuracy and no more halftone patches than previously proposed models such as the Duplex ClapperYule model and the Yule-Nielsen model extended to transmission. The model relies on the separation of the printing support into two sublayers, each one containing the inks of the recto halftone color, or accordingly the ones of the verso halftone color. The model should be limited to printing supports where the inks do not penetrate too much deeply, but a more exhaustive testing is needed to see the precise limitations in practice. The model showed its performance when multiview images are produced from layouts computed with it. Since these prints rely on color matching between different rectoverso halftones, the quality of the displayed images directly depends on the accuracy of the model. 


\section{References}

1. V. Babaei, R.D. Hersch, "Yule-Nielsen based multi-angle reflectance prediction of metallic halftones," Proc. SPIE 9395, paper 93950H (2015).

2. P. Pjanic, R.D.Hersch, "Specular color imaging on a metallic substrate," Proc. IS\&T 21st Color and Imaging Conference, 61-68 (2013).

3. M. Hébert, S. Mazauric, T. Fournel, "Spectral mixing approach for modeling the coloration of surfaces," Workshop on Information Optics, Kyoto, Japan, 1-5 June 2015.

4. J. Machizaud, M. Hébert, "Spectral transmittance model for stacks of transparencies printed with halftone colors," Proc. SPIE 8292, Paper 829240 (2012).

5. M. Hébert, R.D. Hersch, "Reflectance and transmittance model for recto-verso halftone prints: spectral predictions with multi-ink halftones," J. Opt. Soc. Am. A 26, 356-364 (2009).

6. M. Hébert, R.D. Hersch, "Yule-Nielsen based recto-verso color halftone transmittance prediction model," Appl. Opt. 50, 519-525 (2011).

7 Hersch, R.D. and Crété, F., "Improving the Yule-Nielsen modified spectral Neugebauer model by dot surface coverages depending on the ink superposition conditions," Proc.SPIE 5667, 434-445 (2005).

8 S. Mazauric, M. Hebert, L. Simonot, T. Fournel, "Two-flux transfer matrix model for predicting the reflectance and transmittance of duplex halftone prints," J. Opt. Soc. Am. A 31, 27752788 (2014).

9 M. Hébert, F. Emmel, "Two-flux and multiflux matrix models for colored surfaces," in Handbook of Digital Imaging, Mickael Kriss Ed., John Wiley \& Sons, pp. 1233-1277 (2015).

10 Nicodemus, FE, Richmond, JC, Hsia, JJ, Ginsberg, IW, Limperis, T. Geometrical considerations and nomenclature for reflectance, NBS Monograph 160, National Bureau of Standards, Washington, DC, p. 52, 1977.

11 M. Hébert, R.D. Hersch, J.-M. Becker, "Compositional reflectance and transmittance model for multilayer specimens," J. Opt. Soc. Am. A 24, 2628-2644 (2007).

12 P. Kubelka, "New contributions to the optics of intensely light-scattering material, part I," $J$. Opt. Soc. Am. 38, 448-457 (1948).

13 J.L. Saunderson, "Calculation of the color pigmented plastics," J. Opt. Soc. Am. 32, 727-736 (1942).

14 M. Hébert and R.D. Hersch, "A reflectance and transmittance model for recto-verso halftone prints," J. Opt. Soc. Am. A 22, 1952-1967 (2006).

15 F. R. Clapper and J. A. C. Yule, "The Effect of Multiple Internal Reflections on the Densities of Halftone Prints on Paper", J. Opt. Soc. Am. 43, 600-603 (1953).

16. M. Hébert, R.D. Hersch, "Review of spectral reflectance prediction models for halftone prints: calibration, prediction and performance," Color Res. Appl., paper 21907 (2014).

17 G. Wyszecki, and W. S. Stiles, Color Science: Concepts and Methods, Quantitative Data and Formulae, 2nd Ed., Wiley, 1982.

18 M. Hébert, R.D. Hersch, "Classical Print Reflection Models: A Radiometric Approach," J. Im. Sci. Technol. 48, 363-374 (2004).

19 M. Hébert, P. Emmel, "Two-flux and multiflux matrix models for colored surfaces" in Handbook of Digital Imaging, Ed. Mickael Kriss, Wiley, New York, 1233-1277 (2015).

20 N. Dalloz, S. Mazauric, T. Fournel, M. Hébert, "How to design a recto-verso print displaying different images in various everyday-life lighting conditions" IS\&T Electronic Imaging Symposium, Materials appearance (Burlingame, USA, 30 January-2 February 2017). 